\title{
A comparison of Streptococcus agalactiae septic arthritis and non-Streptococcus agalactiae septic arthritis
}

\author{
Victor Tzong-Jing Wang ${ }^{1}$, MBBS, FRCSEd, Jiong Hao Tan $^{1}$, MBBS, MRCs, Leon Han Pay ${ }^{1}$, Tianyi Wu ${ }^{1}$, \\ Liang Shen ${ }^{2}$, BStats, PhD, Gavin Kane $\underline{O ’ N e i l l}^{1}$, FRCSGI, Veerasingam Prem Kumar $^{1}$, MBBS, FRCSEd
}

INTRODUCTION Streptococcus agalactiae (Group B Streptococcus, GBS) is an uncommon cause of septic arthritis in the adult population. In recent years, there has been an increase in the incidence of GBS septic arthritis. This study aims to compare the clinical presentation, investigations, microbiology and outcome of management in patients with GBS and non-GBS septic arthritis.

METHODS Retrospective review of hospital surgical records was done to identify all patients treated surgically at our institution from January 2011 to January 2016 for primary septic arthritis. Patients were categorised into two groups: those with culture-proven GBS septic arthritis and those with causative pathogens that were not GBS. Patients who were medically unfit for surgical intervention as well as those who declined interventional procedures were excluded from the study.

RESULTS A total of 83 patients were included in the study: 62 (74.7\%) had non-GBS septic arthritis and 21 (25.3\%) had GBS septic arthritis. Patients with GBS septic arthritis were more likely to have polyarticular involvement $(p<0.001)$ and involvement of less common sites such as the elbow joint. They were also more likely to have elevated inflammatory markers (C-reactive protein $>150 \mathrm{mg} / \mathrm{L} ; \mathrm{p}=0.017)$ and positive blood cultures $(p=0.02)$, and were typically healthy adults with no medical comorbidities $(p=0.012)$.

CONCLUSION Patients with GBS septic arthritis were more likely to present with polyarticular involvement, positive blood cultures and higher levels of C-reactive protein on admission, and tended to be healthier individuals with no medical comorbidities.

Keywords: bacterial infection, Group B Streptococcus, polyarticular, septic arthritis, Streptococcus agalactiae

\section{INTRODUCTION}

Septic arthritis is an orthopaedic emergency that has potentially severe complications, such as sepsis and limb loss, and an estimated mortality rate of $11 \%$. $^{(1)}$ It generally presents as monoarticular, with polyarticular involvement being less common. Staphylococcus aureus and Streptococcus species are the usual causative organisms. ${ }^{(2-4)}$ Streptococcus species can be broadly classified into two key groups; alpha-haemolytic $(\alpha$-haemolytic) and beta-haemolytic ( $\beta$-haemolytic) streptococci. $\beta$-haemolytic streptococci can be further subdivided into Group A Streptococcus and Group B Streptococcus (GBS). The latter is also known as Streptococcus agalactiae (S. agalactiae). Generally, streptococcal infections (all sites) are very common. The main causative micro-organisms are usually $\alpha$-haemolytic streptococci and $\beta$-haemolytic Group A streptococci. On the other hand, $\beta$-haemolytic GBS are most often associated with severe infections in neonates, pregnant women and immunocompromised hosts such as the elderly population. ${ }^{(5)}$

However, in more recent times, some studies have observed that GBS has become an increasingly common pathogen in adult orthopaedic patients ${ }^{(6-1)}$ and that the incidence of $S$. agalactiaeassociated septic arthritis is rising. ${ }^{(6-8,10)}$ The 2015 outbreak of GBSassociated infections in Singapore sparked nationwide interest in this pathogen and its associated infections. Not surprisingly, we also noticed a rise in the number of GBS infections as well as a corresponding increase in GBS-related septic arthritis here, particularly in 2015. In this study, we sought to study the clinical, epidemiologic characteristics and inpatient outcomes of patients with GBS septic arthritis compared to those with non-GBS septic arthritis.

\section{METHODS}

This study was conducted at National University Hospital (NUH), Singapore, a 1,200-bed tertiary acute care hospital located in western Singapore. The institution contains 41 specialised outpatient clinics/centres with an additional 19 specialised service centres. NUH has strong research and teaching affiliations with the National University of Singapore and is one of Singapore's major tertiary referral centres, serving up to one-fifth of the population.

We retrospectively reviewed the hospital surgical records of all 83 patients who were treated surgically for primary septic arthritis from January 2011 to January 2016. Septic arthritis was defined as the presence of positive synovial fluid culture from the affected joint. In the same time period, patients who were treated non-surgically (i.e. managed purely with antibiotics and/ or had needle aspiration of intra-articular pus) were excluded from this study. $68(81.9 \%)$ patients were treated with open surgical drainage and debridement, and the remaining 15 (18.1\%) patients were treated with arthroscopic debridement and washout

${ }^{1}$ Department of Orthopaedic Surgery, National University Hospital, ${ }^{2}$ Biostatistics Unit, National University of Singapore, Singapore

Correspondence: Dr Tzong-Jing Victor Wang, Registrar, Department of Orthopaedic Surgery, National University Hospital, 5 Lower Kent Ridge Road, Singapore 119074. victorwang80@gmail.com 
of the affected joint, followed by completion of an appropriate antibiotics regime recommended by the infectious disease physicians. All patients were referred to and co-managed with physicians from the infectious disease department. Empirical antibiotics were commenced once the diagnosis of septic arthritis was made and then changed appropriately once the identities of the causative organisms were known. Following the advice of the infectious disease physicians, the antibiotics were continued until a successful outcome was attained, as indicated by complete clinical resolution of symptoms and downtrending of inflammatory markers.

The following parameters were obtained from hospital inpatient records: age, ethnicity, gender, type of comorbidities, blood, biochemical markers (including C-reactive protein [CRP] levels on admission), radiographic investigations, antibiotics treatment, type of surgical procedures undergone and outcome of patients during both inpatient as well as outpatient follow-up visits. The study cohort was divided into two groups, patients with culture-proven $S$. agalactiae (i.e. GBS) septic arthritis and patients with septic arthritis secondary to other pathogens. Patients who were medically unfit or moribund for joint aspiration or surgery, and those who refused to give consent for any form of interventional procedure were automatically excluded from this study, because pure medical treatment of septic arthritis could have skewed our observations.

Statistical analysis was performed using IBM SPSS Statistics version 22.0 (IBM Corp, Armonk, NY, USA). Wilcoxon ranksum test and Mann-Whitney $U$ test were used for continuous variables, while chi-square test and Fisher's exact test were used for categorical variables. Logistic regression was performed on the significant variables identified. A p-value $<0.05$ was considered to be statistically significant.

\section{RESULTS}

A total of 83 patients with proven septic arthritis were treated between January 2011 and January 2016. Of these patients, $62(74.7 \%)$ had non-GBS septic arthritis, while 21 (25.3\%) had GBS septic arthritis. Table I summarises the microbiological identification of the non-GBS cases. Of the 21 patients diagnosed with GBS septic arthritis, 15 (71.4\%) were diagnosed between January 2015 and January 2016. This made up 38.5\% of the 39 septic arthritis cases treated in this time period, which is significantly more than during the period of January $2011-$ December 2014, when there were 6 (13.6\%) cases of GBS septic arthritis out of 44 cases $(p=0.009)$.

Taking into account the ten patients in the same admission who died due to septic arthritis as a primary or secondary cause, the remaining 73 patients were followed up over a mean period of 8.13 (range 2-58) months. The wide range, after excluding the deceased patients, was mainly due to patients who were on longterm follow-up for other unrelated chronic orthopaedic problems such as concomitant osteoarthritis, fractures in other regions of the musculoskeletal system and gouty arthritis.

Table II summarises the demographics, comorbidities and clinical presentation of the two groups of patients. No significant
Table I. Microbiological identification of the non-Group B Streptococcus cases $(n=62)$.

\begin{tabular}{|ll|}
\hline Cause & No. \\
\hline Staphylococcus aureus & 29 \\
\hline Edwardsiella tarda & 1 \\
\hline Gonococcus & 2 \\
\hline Streptococcus pyogenes & 2 \\
\hline Streptococcus dysgalactiae & 12 \\
\hline Pseudomonas & 3 \\
\hline Escherichia coli & 2 \\
\hline Coagulase-negative Staphylococcus aureus & 4 \\
\hline Methicillin-resistant Staphylococcus aureus & 3 \\
\hline Prevotella & 1 \\
\hline Klebsiella pneumoniae & 1 \\
\hline Salmonella & 1 \\
\hline Streptococcus pneumoniae & 1 \\
\hline
\end{tabular}

Table II. Patient demographics, comorbidities and presentation.

\begin{tabular}{|c|c|c|c|}
\hline \multirow[t]{2}{*}{ Parameter } & \multicolumn{2}{|c|}{ No. (\%) } & \multirow[t]{2}{*}{ p-value } \\
\hline & $\begin{array}{l}\text { Non-GBS } \\
(n=62)\end{array}$ & $\begin{array}{l}\text { GBS } \\
(n=21)\end{array}$ & \\
\hline 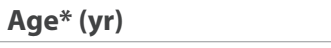 & 63.0 & 56.1 & 0.661 \\
\hline Gender & & & 0.553 \\
\hline Male & $46(74.2)$ & $16(76.2)$ & \\
\hline Female & $16(25.8)$ & $5(23.8)$ & \\
\hline Ethnicity & & & 0.154 \\
\hline Chinese & $39(62.9)$ & $18(85.7)$ & \\
\hline Malay & $16(25.8)$ & $1(4.8)$ & \\
\hline Others & $7(11.3)$ & $2(9.5)$ & \\
\hline \multicolumn{4}{|l|}{ Comorbidity } \\
\hline Diabetes mellitus & $21(33.9)$ & $6(28.6)$ & 0.435 \\
\hline Hypertension & $35(56.5)$ & $5(23.8)$ & 0.009 \\
\hline Hyperlipidaemia & $27(43.5)$ & $3(14.3)$ & 0.013 \\
\hline Ischaemic heart disease & $15(24.2)$ & $1(4.8)$ & 0.043 \\
\hline Renal impairment & $21(33.9)$ & $0(0)$ & 0.001 \\
\hline$\geq 1$ comorbidity & $43(69.4)$ & $8(38.1)$ & 0.012 \\
\hline \multicolumn{4}{|l|}{ Joint involvement/site } \\
\hline No. of joints involved & & & $<0.001$ \\
\hline Single & $58(93.5)$ & $11(52.4)$ & \\
\hline Multiple & $4(6.5)$ & $10(47.6)$ & \\
\hline Knee & $38(61.3)$ & $15(71.4)$ & 0.287 \\
\hline Shoulder & $13(21.0)$ & $6(28.6)$ & 0.331 \\
\hline Elbow & $1(1.6)$ & $3(14.3)$ & 0.048 \\
\hline Ankle & $7(11.3)$ & $5(23.8)$ & 0.147 \\
\hline Others & $7(11.3)$ & $6(28.6)$ & 0.067 \\
\hline
\end{tabular}

*Data presented as mean. GBS: Group B Streptococcus

differences were found in the age, gender or ethnicity of the patients. Among patients with GBS septic arthritis, there was a significantly lower incidence of hypertension, hyperlipidaemia, ischaemic heart disease, renal impairment and the presence of one or more of the above comorbidities. Generally, the knee and the shoulder were the most common sites of infection for both groups of patients, although 
patients with GBS septic arthritis were more likely to present in less common sites such as the elbow joint (14.3\% vs. $1.6 \%$; $p=0.048)$. Patients with GBS septic arthritis were also more likely to present with multiple joint involvement as compared to patients with nonGBS septic arthritis (47.6\% vs. 6.5\%; $p<0.001)$.

As shown in Table III, the incidence of positive blood cultures was significantly higher in patients with GBS septic arthritis as compared to patients with non-GBS septic arthritis (81.0\% vs. $41.9 \% ; p=0.02$ ). In addition, GBS isolates cultured from the synovial fluid of five patients with GBS septic arthritis were further characterised genetically. It was found that the same sequence type (Serotype III ST283) of $S$. agalactiae was cultured from the synovial fluid of all five patients. From hospital admission and inpatient records, we observed that the CRP levels (on admission) of patients with GBS septic arthritis were more likely to be higher on presentation (Table III). An elevated CRP level > $150 \mathrm{mg} / \mathrm{L}$ was present in $20(95.2 \%)$ out of 21 patients with GBS septic arthritis, as compared to $44(71.0 \%)$ out of 62 non-GBS patients $(p=0.017)$. Other investigations in these two groups of patients had no significant differences.

All 83 patients included in the study underwent surgical intervention in the form of either open arthrotomy debridement and washout $(81.9 \%, \mathrm{n}=68)$ or arthroscopic debridement and

Table III. Inpatient outcomes and investigations.

\begin{tabular}{|c|c|c|c|}
\hline \multirow[t]{2}{*}{ Parameter } & \multicolumn{2}{|c|}{ No. (\%) } & \multirow[t]{2}{*}{ p-value } \\
\hline & $\begin{array}{l}\text { Non-GBS } \\
(n=62)\end{array}$ & $\begin{array}{l}\text { GBS } \\
(n=21)\end{array}$ & \\
\hline \multicolumn{4}{|l|}{ Investigation } \\
\hline Blood culture & $26(41.9)$ & $17(81.0)$ & 0.02 \\
\hline $\mathrm{CRP}>150 \mathrm{mg} / \mathrm{L}$ & $44(71.0)$ & $20(95.2)$ & 0.017 \\
\hline \multicolumn{4}{|l|}{ Outcome } \\
\hline Length of treatment* $(w k)$ & 5.95 & 7.67 & 0.03 \\
\hline Length of stay* (day) & 21 & 25 & 0.255 \\
\hline $\mathrm{HDU} / \mathrm{ICU}$ & $29(46.8)$ & $5(23.8)$ & 0.054 \\
\hline Death & $10(16.1)$ & $0(0)$ & 0.044 \\
\hline $2011-2014^{\dagger}$ & $4 / 38(10.5)$ & $0 / 6(0)$ & 0.544 \\
\hline $2015-2016^{+}$ & $6 / 24(25.0)$ & $0 / 15(0)$ & 0.041 \\
\hline Limb loss & $3(4.8)$ & $0(0)$ & 0.412 \\
\hline Multiple operations & $16(25.8)$ & $9(42.9)$ & 0.117 \\
\hline Readmission & $8(12.9)$ & $2(9.5)$ & 0.511 \\
\hline
\end{tabular}

*Data presented as mean. †Percentages are calculated according to the totals provided. CRP: C-reactive protein; GBS: Group B Streptococcus; HDU: high dependency unit; ICU: intensive care unit washout (18.1\%, $\mathrm{n}=15$ ) of the affected joint. 5 (23.8\%) out of 21 patients with GBS septic arthritis underwent the arthroscopic procedure, as compared to $10(16.1 \%)$ out of 62 patients with non-GBS septic arthritis. In terms of differences in the type of surgical intervention elected and performed, we did not find any significant difference between the two groups of patients $(p=0.313)$. The mean duration of treatment for the entire cohort of patients was 6.39 (median 6) weeks. When the two groups were compared, patients with GBS septic arthritis required a mean of 7.67 (95\% confidence interval [CI] 6.58-8.76) weeks of treatment, which was significantly longer than those with non-GBS septic arthritis, who required $5.95(95 \% \mathrm{Cl} 5.27-6.63)$ weeks $(p=0.03)$.

Outcomes studied included length of stay, need for monitoring at the high dependency unit (HDU) or intensive care unit (ICU), number of operations undergone, need for readmission within 30 days, limb loss, and death (Table III). We found that patients with GBS septic arthritis had a significantly lower mortality rate, with no patient deaths as compared to 10 (16.1\%) out of 62 patients with non-GBS septic arthritis $(p=0.044)$. We further stratified the mortality rates of the two groups by time period (i.e. 2011-2014 vs. 2015-2016, the ST283 GBS outbreak period) and found that there were significantly higher mortality rates for patients with non-GBS septic arthritis only during the 2015-2016 period $(p=0.041)$. There were no other significant differences in terms of the number of operations required, readmission rates, amputation rates and need for HDU/ICU care. On multivariable regression, we found that the presence of positive blood cultures, multiple joint involvement and absence of medical comorbidities were the only variables significantly associated with GBS septic arthritis (Table IV).

\section{DISCUSSION}

GBS, also known as $S$. agalactiae, is a type of Gram-positive bacteria and a common commensal bacterium of bowel and genital tract flora. Known transmission routes include sexual contact with colonised individuals, as well as vertical transmission from mother to neonate. ${ }^{(12,13)}$ Colonisation usually does not lead to disease. Invasive GBS infections in healthy adults are not as common compared to severe infections in pregnant women, neonates, the elderly and immunocompromised individuals. . $5,12,14-19)^{2}$

Previous studies have described GBS causing bovine mastitis ${ }^{(20)}$ and disease in fish. ${ }^{(21)}$ However, our understanding of the links between the routes and likelihood of interspecies transmission and invasive disease in humans remains lacking.

Table IV. Differences in clinical features and comorbidities between Group B Streptococcus (GBS) septic arthritis and non-GBS septic arthritis.

\begin{tabular}{|c|c|c|c|c|}
\hline \multirow[t]{2}{*}{ Parameter } & \multicolumn{2}{|c|}{ No. (\%) } & \multicolumn{2}{|c|}{ Multivariate model } \\
\hline & Non-GBS $(n=62)$ & GBS $(n=21)$ & Adjusted OR (95\% Cl) & p-value \\
\hline Elbow & $1(1.6)$ & $3(14.3)$ & $6.27(0.007-550.657)$ & 0.596 \\
\hline Multiple joint involvement & $4(6.5)$ & $10(47.6)$ & $25.69(2.126-310.388)$ & 0.011 \\
\hline Bacteraemia* & $26(41.9)$ & $17(81.0)$ & $12.225(1.914-78.072)$ & 0.008 \\
\hline CRP > 150 mg/L & $44(71.0)$ & $20(95.2)$ & 4.379 (0.398-48.213) & 0.228 \\
\hline Comorbidities $\geq 1$ & $43(69.4)$ & $8(38.1)$ & $0.141(0.026-0.750)$ & 0.022 \\
\hline
\end{tabular}

*Defined as the presence of positive blood cultures. Cl: confidence interval; CRP: C-reactive protein; OR: odds ratio 
One study has drawn attention to a possible link in interspecies transmission of GBS from animals to humans, resulting in GBS colonisation in healthy adults. ${ }^{(13)}$ Foxman et al observed an increased risk of GBS colonisation (capsular subtype la and Ib) with fish consumption in healthy adults. ${ }^{(13)}$ However, the study only looked at the association between GBS colonisation and fish consumption, and hence could not establish a link between fish consumption and GBS infections in humans.

The recent outbreak of GBS-associated infections in Singapore sparked a widespread interest in this pathogen and its associated infections. Two recent local studies, conducted in the aftermath of the outbreak, identified a strong association between consumption of raw or undercooked fish and GBS Serotype III ST283 bacteraemia/ invasive infections in men and non-pregnant women. ${ }^{(22,23)}$ Their findings were further supported by the presence of GBS Serotype III ST283, the particular sequence associated with invasive disease in human beings, in fish samples tested by the Agri-Food and Veterinary Authority of Singapore and National Environment Agency. Tan et al ${ }^{(22)}$ retrospectively studied a cohort of 40 case patients with GBS bacteraemia, comparing them with 58 case controls, and found that the odds ratios of GBS bacteraemia among patients who ate raw fish were 8.58 times that of those who had not eaten raw fish. Further genetic testing of bacteria isolates from case patients showed that all patients who consumed raw fish $(\mathrm{n}=19)$ had Serotype III ST283, whereas case patients who did not eat raw fish had Serotypes Ia, II, III, VI and VII. The authors also found that the mean age of case patients infected with ST283 was significantly younger than those with non-ST283 (57.4 years vs. 68.6 years; $\mathrm{p}=0.014)$. In addition, the authors reported that a significantly lower proportion of patients infected with ST283 reported having underlying medical conditions, compared to non-ST283 patients (56\% vs. $100 \%$; $p=0.003) .{ }^{(22)}$ A second prospective case control study of 22 case patients with GBS bacteraemia or infections, conducted by Rajendram et al ${ }^{(23)}$ during the epidemiologic weeks of the outbreak, drew similar observations. The authors found that patients infected with ST283 tended to be younger than those with non-ST283 (median age 59.4 years vs. 74.0 years; $p=0.033$ ) and that they were less likely to have pre-existing medical conditions (33.3\% vs. $84.6 \% ; p=0.014)$. Both studies also observed a sharp drop in the number of invasive GBS cases after a public advisory was issued in July 2015 to halt the sales of certain species of raw freshwater fish (Asian big head carp and snakehead fish). This lends substantial support to the association between raw fish consumption and invasive GBS infections.

Staphylococcus aureus is the most common micro-organism found in septic arthritis, accounting for up to $60 \%$ of cases, ${ }^{(2-4,24,25)}$ followed by Streptococci species. In recent years, however, various studies have highlighted GBS as an increasingly important pathogen in adult septic arthritis. ${ }^{(6-8,10)}$ As early as 1984, Laster et $\mathrm{al}^{(6)}$ first alluded to the possible emergence of GBS as a pathogen in septic arthritis. They observed that for decades, only 18 cases had been described (eight cases after 1976), and that their two cases of GBS septic arthritis presenting within the same year should therefore not be attributed to pure coincidence. Findings from a more recent study by Louthrenoo et $\mathrm{a}^{(8)}$ seemed to support this theory. The authors reported an incidence of $15.57 \%$ for S. agalactiae septic arthritis in patients treated for the infection between 1990 and 2010, of which 90\% were diagnosed between 2008 and 2010. However, the authors were unable to find a cause for the increased infection rates. Instead, they speculated that it was related to the change in climate.

To our knowledge, no studies to date have specifically looked at the impact of the recent GBS outbreak on joint infections. In our study, about one-quarter of the septic arthritis cases were caused by GBS. Among these patients, those diagnosed in the January 2015-January 2016 period made up 38.5\% of the septic arthritis cases treated during this period, significantly more than the proportion of $13.7 \%$ during the January 2011-December 2014 period $(p=0.009$ ). This seems to concur with previous findings in the literature. In addition, all five patients who underwent further serotyping of the isolated bacteria yielded the same serotype (Serotype III ST283) of $S$. agalactiae. Our results are consistent with the findings of the Singapore Streptococcus agalactiae Working Group, which identified the same serotype of $S$. agalactiae in local patients with GBS infections as part of the outbreak investigation. ${ }^{(26)}$

Septic arthritis usually presents with monoarticular involvement. In our study, we observed that only slightly more than half $(52.4 \%)$ of all GBS cases presented with single joint involvement compared to septic arthritis from other causes, of which $93.5 \%$ were monoarticular $(p=0.011)$. The most common sites of septic arthritis in both sets of patients were the knee and the shoulder, similar to the findings of Nolla et $\mathrm{al}^{(7)}$ and Louthrenoo et al. ${ }^{(8)}$ However, compared to patients with non-GBS septic arthritis, a higher proportion of patients with GBS septic arthritis presented with less typical primary sites of infection such as the elbow joint $(14.3 \%$ vs. $1.6 \%$, $p=0.048)$. In our multivariate analysis, however, this finding was not statistically significant $(\mathrm{p}=$ 0.596). Although $S$. agalactiae is known to affect the small joints of the body, such as the sternoclavicular joint and wrist joint, ${ }^{(27,28)}$ we did not detect any significant difference between the two groups of patients with regard to small joint involvement $(p=0.067)$.

Septic arthritis is a condition that usually arises in the very young or old, patients with underlying joint disease (e.g. rheumatoid arthritis), individuals with underlying medical illnesses (e.g. diabetes mellitus) and immunocompromised patients. ${ }^{(29,30)}$ In previous studies, up to two-thirds of patients with $S$. agalactiae infections had underlying conditions that predisposed them to infection. ${ }^{(7,31)}$ These conditions included cancer, diabetes mellitus, systemic inflammatory disease (e.g. rheumatoid arthritis) and chronic liver disease, as well as diseases requiring corticosteroid therapy. Some authors have theorised that age and immunosuppression are important predisposing factors. ${ }^{(7,27-29,31)}$ This was not so for our cohort, where logistic regression showed that GBS septic arthritis tended to affect healthier individuals with no significant comorbidities $(p=0.022)$. This difference from previous studies highlights the fact that the particular virulence factors of this disease still remain unknown.

No studies to date have managed to produce significant diagnostic results capable of differentiating between GBS and 
non-GBS septic arthritis. Our study identified some differences between the two entities. We observed that $81.0 \%$ of patients with GBS septic arthritis had positive blood cultures as compared to $41.9 \%$ of patients with non-GBS septic arthritis, which was significant on logistic regression $(p=0.008)$. This finding was comparable to various studies that also reported similar associations between GBS infections and bacteraemia. ${ }^{(8,19,22)}$ In addition, our results showed that patients with GBS septic arthritis were more likely to have significantly higher CRP values, with $95.2 \%$ of these patients having CRP $>150 \mathrm{mg} / \mathrm{L}$. In contrast, only $71.0 \%$ of patients with septic arthritis from non-GBS microorganisms had CRP $>150 \mathrm{mg} / \mathrm{L}$ on univariate analysis $(p=0.017)$. However, on multivariate logistic regression, this difference was not statistically significant $(p=0.228)$.

This study included only patients who were treated surgically and excluded those who were managed purely with antibiotics and/or had needle aspiration of intra-articular pus. The intention was to exclude patients who were moribund or medically too unwell for surgery, sometimes due to the severity of the disease. Some authors might argue that regardless of disease severity, compared to appropriate initial surgical management, managing septic arthritis non-surgically (i.e. needle aspiration and/or antibiotics) does not affect the outcome. Indeed, a study by Goldenberg et al ${ }^{(32)}$ comparing needle aspiration to surgery as an initial mode of treatment in septic arthritis found that neither treatment was superior to the other. However, at our institution, adequate debridement and drainage of the infective focus, which can only be achieved via surgical means, remains the gold standard in successful management of pyogenic joint infections.

Both groups of patients underwent surgical treatment in the form of either open arthrotomy washout or arthroscopic washout, and there was no difference in the type of surgical intervention performed. However, we did observe that patients suffering from GBS septic arthritis required a significantly longer duration of treatment compared to patients with non-GBS septic arthritis (7.67 weeks vs. 5.95 weeks; $p=0.003)$. This difference in treatment duration can potentially confound intermediate to long-term outcomes rather than inpatient or short-term clinical outcomes (i.e. mortality rates, limb loss, length of hospitalisation, HDU/ICU care, limb loss, requirement of multiple surgeries and readmission rates), which were the main focus of our study.

Our study had several limitations. Firstly, the large diversity of causative organisms within the non-GBS group required the assumption that the septic arthritis caused by the various microorganisms has similar characteristics. However, we do not have conclusive evidence on this, and our numbers within the group were too small to perform further subgroup analysis. The control arm remains too diverse to make definitive conclusions about the virulence factors of the bacteria and their role in joint infections. Next, because our study straddles a unique time when there was an outbreak of invasive GBS (Serotype III ST283) infections, and 15 out of 21 cases were in the 2015-2016 period, we recognise that our results could be confined to this particular outbreak sequence type and possibly cannot be extrapolated to GBS septic arthritis in general. In addition, the lack of isolate collection and genetic characterisation of all cases of GBS-associated septic arthritis did not allow us to examine the particular risk and virulence factors of the different GBS strains. Lastly, as this was a retrospective study with a relatively short mean follow-up duration of 8.13 months, we were unable to investigate long-term outcome measures such as late recurrence rates and long-term complications such as risk of early osteoarthritis. A follow-up study of this cohort with a longer follow-up duration would hopefully allow us to draw more conclusions about the long-term sequelae of GBS septic arthritis.

This study has yielded several interesting observations on the differences between the two groups of patients. However, these findings are by no means conclusive. The management of all kinds of infections, and not just orthopaedic-specific sepsis, hinges upon understanding the complex interplay among host factors, specific micro-organism virulence factors and treatmentrelated specifics. Further follow-up and research in the form of prospective matched case-control studies are necessary to help orthopaedic physicians to better manage septic arthritis.

In conclusion, patients with GBS septic arthritis are more likely to be healthy individuals with absence of predisposing comorbidities. They also tend to present with multiple joint involvement and positive blood cultures. In healthy patients presenting with septic arthritis and $S$. agalactiae bacteraemia, care must be taken to exclude multiple sites of septic arthritis. They should then be treated with appropriate antibiotics as well as surgical drainage of all affected sites.

\section{REFERENCES}

1. Gupta MN, Sturrock RD, Field M. A prospective 2-year study of 75 patients with adult-onset septic arthritis. Rheumatology (Oxford) 2001; 40:24-30.

2. Weston VC, Jones AC, Bradbury N, Fawthrop F, Doherty M. Clinical features and outcome of septic arthritis in a single UK Health District 1982-1991. Ann Rheum Dis 1999; 58:214-9.

3. Dubost JJ, Soubrier M, De Champs C, et al. No changes in the distribution of organisms responsible for septic arthritis over a 20 year period. Ann Rheum Dis 2002; 61:267-9.

4. Le Dantec L, Maury F, Flipo RM, et al. Peripheral pyogenic arthritis. A study of one hundred seventy-nine cases. Rev Rhum Engl Ed 1996; 63:103-10.

5. Kothari NJ, Morin CA, Glennen A, et al. Invasive group B streptococcal disease in the elderly, Minnesota, USA, 2003-2007. Emerg Infect Dis 2009; 15:1279-81.

6. Laster AJ, Michels ML. Group B streptococcal arthritis in adults. Am J Med 1984; 76:910-5.

7. Nolla JM, Gómez-Vaquero C, Corbella X, et al. Group B streptococcus (Streptococcus agalactiae) pyogenic arthritis in nonpregnant adults. Medicine (Baltimore) 2003; 82:119-28.

8. Louthrenoo W, Kasitanon N, Wangkaew S, et al. Streptococcus agalactiae: an emerging cause of septic arthritis. J Clin Rheumatol 2014; 20:74-8.

9. Jenkins PJ, Clement ND, Gaston P, et al. Invasive group B streptococcal disease in an orthopaedic unit. J Hosp Infect 2010; 76:231-3.

10. Binard A, Devauchelle V, Goulesque K, Jousse S, Saraux A. Group B streptococcal arthritis. Joint Bone Spine 2006; 73:465-8.

11. Smith EM, Khan MA, Reingold A, Watt JP. Group B streptococcus infections of soft tissue and bone in California adults, 1995-2012. Epidemiol Infect 2015; 143:3343-50.

12. Schuchat A. Group B streptococcus. Lancet 1999; 353:51-6.

13. Foxman B, Gillespie BW, Manning SD, Marrs CF. Risk factors for group B streptococcal colonization: potential for different transmission systems by capsular type. Ann Epidemiol 2007; 17:854-62.

14. Tazi A, Morand PC, Réglier-Poupet $\mathrm{H}$, et al. Invasive group B streptococcal infections in adults, France (2007-2010). Clin Microbiol Infect 2011; 17:1587-9.

15. Skoff TH, Farley MM, Petit S, et al. Increasing burden of invasive group B streptococcal disease in nonpregnant adults, 1990-2007. Clin Infect Dis 2009; 49:85-92.

16. Falagas ME, Rosmarakis ES, Avramopoulos I, Vakalis N. Streptococcus agalactiae infections in non-pregnant adults: single center experience of a growing clinical 
problem. Med Sci Monit 2006; 12:CR447-51.

17. Phares CR, Lynfield R, Farley MM, et al; Active Bacterial Core surveillance/ Emerging Infections Program Network. Epidemiology of invasive group B streptococcal disease in the United States, 1999-2005. JAMA 2008 299:2056-65

18. Blancas D, Santin M, Olmo M, et al. Group B streptococcal disease in nonpregnant adults: incidence, clinical characteristics, and outcome. Eur J Clin Microbiol Infect Dis 2004; 23:168-73.

19. Tan K, Wijaya L, Chiew HJ, et al. Diffusion-weighted MRI abnormalities in an outbreak of Streptococcus agalactiae serotype III, multilocus sequence type 283 meningitis. J Magn Reson Imaging 2017; 45:507-14.

20. Oliveira IC, de Mattos MC, Pinto TA, et al. Genetic relatedness between group B streptococci originating from bovine mastitis and a human group B Streptococcus type $\mathrm{V}$ cluster displaying an identical pulsed-field gel electrophoresis pattern. Clin Microbiol Infect 2006; 12:887-93.

21. Evans JJ, Klesius PH, Pasnik DJ, Bohnsack JF. Human Streptococcus agalactiae isolate in Nile Tilapia (Oreochromis niloticus). Emerg Infect Dis 2009; 15:774-6

22. Tan S, Lin Y, Foo K, et al. Group B Streptococcus serotype III sequence type 283 bacteremia associated with consumption of raw fish, Singapore. Emerg Infect Dis 2016; 22:1970-3.

23. Rajendram P, Mar Kyaw W, Leo YS, et al. Group B Streptococcus sequence type 283 disease linked to consumption of raw fish, Singapore. Emerg Infect Dis $2016 ; 22: 1974-7$.
24. Chaiwarith R, Jullaket W, Bunchoo M, et al. Streptococcus agalactiae in adults at Chiang Mai University Hospital: a retrospective study. BMC Infect Dis 2011; 11:149.

25. Rosenthal J, Bole GG, Robinson WD. Acute nongonococcal infectious arthritis. Evaluation of risk factors, therapy, and outcome. Arthritis Rheum 1980; 23:889-97.

26. Mehershahi KS, Hsu LY, Koh TH, Chen SL. Complete genome sequence of Streptococcus agalactiae serotype III, multilocus sequence type 283 strain SGM1. Genome Announc 2015; 3:e01188-15.

27. Carrascosa M, Pascual F, Corrales A, et al. Septic sternoclavicular arthritis caused by group B Streptococcus: case report and review. Clin Infect Dis 1996; 22:579-80.

28. Stark RH. Group B beta-hemolytic streptococcal arthritis and osteomyelitis of the wrist. J Hand Surg Am 1987; 12:296-9.

29. Ross JJ. Septic arthritis. Infect Dis Clin North Am 2005; 19:799-817.

30. Mathews CJ, Weston VC, Jones A, Field M, Coakley G. Bacterial septic arthritis in adults. Lancet 2010; 375:846-55.

31. Ho CM, Chi CY, Ho MW, et al. Clinical characteristics of group B streptococcus bacteremia in non-pregnant adults. J Microbiol Immunol Infect 2006; 39:396-401.

32. Goldenberg DL, Brandt KD, Cohen AS, Cathcart ES. Treatment of septic arthritis: comparison of needle aspiration and surgery as initial modes of joint drainage. Arthritis Rheum 1975; 18:83-90. 